\title{
A simple clinical colitis activity index
}

\author{
R S Walmsley, R C S Ayres, R E Pounder, R N Allan
}

\begin{abstract}
Background-The appropriate medical treatment of patients with ulcerative colitis is determined largely by the severity of symptoms. Hospital assessment of the severity of disease activity includes investigation of laboratory indices and sigmoidoscopic assessment of mucosal inflammation.
\end{abstract}

Aims-To develop a simplified clinical colitis activity index to aid in the initial evaluation of exacerbations of colitis.

Methods-The information for development of the simple index was initially evaluated in 63 assessments of disease activity in patients with ulcerative colitis where disease activity was evaluated using the Powell-Tuck Index (which includes symptoms, physical signs, and sigmoidoscopic appearance). The new index was then further evaluated in 113 assessments in a different group of patients, by comparison with a complex index utilising clinical and laboratory data, as well as five haematological and biochemical markers of disease severity.

Results-The newly devised Simple Clinical Colitis Activity Index, consisting of scores for five clinical criteria, showed a highly significant correlation with the Powell-Tuck Index $(r=0.959, p<0.0001)$ as well as the complex index $(r=0.924$, $p<0.0001)$ and all laboratory markers $(\mathrm{p}=0.0003$ to $\mathrm{p}<0.0001)$.

Conclusions-This new Simple Colitis Activity Index shows good correlation with existing more complex scoring systems and therefore could be useful in the initial assessment of patients with ulcerative colitis.

(Gut 1998;43:29-32)

Inflammatory Bowel

Keywords: ulcerative colitis; disease activity

Disease Study Group,

Royal Free Hospital

School of Medicine,

London, UK

R S Walmsley

R E Pounder

Gastroenterology Unit, Queen Elizabeth

Hospital, Edgbaston,

Birmingham, UK

R C S Ayres

R N Allan

Correspondence to: Dr R N Allan, Queen

Elizabeth Hospital,

Edgbaston,

Birmingham B15 2TH, UK.

Accepted for publication 4 February 1998

Ulcerative proctocolitis is a common disorder with an annual incidence of 10 per 100000 population in the UK. ${ }^{12}$ For the majority of patients it is a chronic relapsing disorder requiring regular clinical follow up. Family practitioners and gastroenterologists are involved in evaluating the severity of exacerbations and the prescription of appropriate medical treatment. Complete assessment of disease activity of ulcerative colitis involves symptomatic evaluation, physical examination, blood tests for laboratory indices, and sigmoidoscopic assessment. However the results of blood tests are not available immediately and sigmoidoscopic assessment may be inappropriate in the home or in a busy family practitioner's clinic.
The aim of this study was to devise an accurate, easily calculated index of disease activity using a small number of clinical criteria. The index should be readily calculated by a family practitioner or physician at an outpatient consultation and should not require physical examination, sigmoidoscopic evaluation, or laboratory indices. The final index could then act as an initial guide to appropriate changes in treatment and be an aid in identifying those patients requiring more detailed assessment.

\section{Methods}

INITIAL DERIVATION OF THE SIMPLE INDEX The Powell-Tuck Activity Score ${ }^{3}$ was used as an established index for evaluating disease activity in order to devise the simple colitis activity index. For all patients all clinical criteria, including findings on physical examination and sigmoidoscopic assessment required to calculate the Powell-Tuck Index (table 1), were recorded together with additional clinical criteria suggested by more recent studies. These additional clinical criteria included nocturnal bowel movement and urgency of defecation, both significantly related to active disease,${ }^{4}$ and the general well being score from the Harvey-Bradshaw simple index of Crohn's disease activity, ${ }^{5}$ as an alternative to the general health question in the Powell-Tuck Index. The category of extracolonic manifestations/ features incorporates arthritis, pyoderma gangrenosum, erythema nodosum and uveitis. The sigmoidoscopic evaluation of the Powell-Tuck Index uses the scoring system of Baron et al. ${ }^{6}$ The sigmoidoscopic and physical examinations were carried out by one observer. The simple clinical score was then devised by adapting the scores given to five clinical criteria to give the best correlation with the Powell-Tuck score.

Ethical approval for the study was given by the Research Ethics Committee of the South Birmingham Health Authority and written consent was obtained from participating patients.
FURTHER VALIDATION OF THE SIMPLE INDEX

The resultant simple clinical colitis activity index was then further evaluated in a different group of patients, with continuing assessment through the course of the disease, by comparison with a complex disease activity index derived from a combination of clinical and laboratory data $^{78}$ (table 2) and with five laboratory markers of disease activity: haemoglobin, haematocrit, platelet count, erythrocyte sedimentation rate, and serum albumin. ${ }^{9} 10$ Patients completed a questionnaire covering the clinical criteria of both indices and the scores were calculated when laboratory data became available. 
Table 1 Scoring system for the Powell-Tuck Index

\begin{tabular}{|c|c|}
\hline Symptoms and signs & Score \\
\hline \multicolumn{2}{|l|}{ Symptoms } \\
\hline \multicolumn{2}{|l|}{ Bowel frequency } \\
\hline $3-6$ & 1 \\
\hline$>6$ & 2 \\
\hline \multicolumn{2}{|l|}{ Stool consistency } \\
\hline Formed & 0 \\
\hline Semi-formed & 1 \\
\hline Liquid & 2 \\
\hline \multicolumn{2}{|l|}{ Abdominal pain } \\
\hline Before/after bowel motions & 1 \\
\hline Prolonged & 2 \\
\hline Anorexia & 1 \\
\hline Nausea/vomiting & 1 \\
\hline \multicolumn{2}{|l|}{ General health } \\
\hline Normal & 0 \\
\hline Slightly impaired & 1 \\
\hline Activities restricted & 2 \\
\hline Unable to work & 3 \\
\hline \multicolumn{2}{|l|}{ Extracolonic manifestations } \\
\hline One/mild & 1 \\
\hline More than one/severe & 2 \\
\hline \multicolumn{2}{|l|}{ Signs } \\
\hline \multicolumn{2}{|l|}{ Abdominal tenderness } \\
\hline Mild & 1 \\
\hline Marked & 2 \\
\hline Rebound & 3 \\
\hline \multicolumn{2}{|l|}{ Body temperature $\left({ }^{\circ} \mathrm{C}\right)$} \\
\hline$<37.1$ & 0 \\
\hline $37.1-38$ & 1 \\
\hline$>38$ & 2 \\
\hline \multicolumn{2}{|l|}{ Blood in stool } \\
\hline Trace & 1 \\
\hline More than trace & 2 \\
\hline \multicolumn{2}{|l|}{ Sigmoidoscopy } \\
\hline Non-haemorrhagic & 0 \\
\hline Friable & 1 \\
\hline Spontaneous bleed & 2 \\
\hline
\end{tabular}

Table 2 Scoring system for the complex integrated disease activity index (Seo et al')

\begin{tabular}{lll}
\hline Variable & Score & Weighting \\
\hline $\begin{array}{l}\text { Bloody stool } \\
\quad \text { Little or none }\end{array}$ & & $\times 60$ \\
$\quad$ Present & 1 & \\
Bowel movements/day & & \\
$\quad \leq 4$ & 1 & \\
$5-7$ & 2 & \\
$\quad \geqslant 8$ & 3 & \\
Erythrocyte sedimentation rate & & $\times 0.5$ \\
$\quad(\mathrm{~mm} / \mathrm{h})$ & & $\times-4$ \\
Haemoglobin $(\mathrm{g} / \mathrm{dl})$ & & $\times-15$ \\
Albumin $(\mathrm{g} / \mathrm{dl})$ & & 200 \\
Constant & &
\end{tabular}

The final score is the total of the resulting values in the right hand column.

Comparison of indices and laboratory data was performed by Spearman rank correlation; the significance taken was $\mathrm{p}<0.05$.

\section{Results}

INITIAL DERIVATION OF THE SIMPLE CLINICAL INDEX

A total of 63 assessments of disease activity were made on 57 patients, comprising 44 outpatients and 19 inpatient events. The median age of the patients was 49.5 years (range 20-84.7); 30 were female. Twenty one patients had total/extensive disease, 15 left sided colitis, nine proctosigmoiditis, and 12 proctitis alone.

There was an increasing positive correlation as the number of clinical criteria included was increased, but in order to keep the clinical index simple only five were included in the final score. The best correlation was obtained between the Powell-Tuck Index and the Simple Clinical Colitis Activity Index using the five
Table 3 Clinical scoring system for the Simple Clinical Colitis Activity Index

\begin{tabular}{ll}
\hline Symptom & Score \\
\hline Bowel frequency (day) & \\
$1-3$ & 1 \\
$4-6$ & 2 \\
$7-9$ & 3 \\
$>9$ & 1 \\
Bowel frequency (night) & 2 \\
$1-3$ & \\
$4-6$ & 1 \\
Urgency of defecation & 2 \\
Hurry & 3 \\
Immediately & \\
Incontinence & 1 \\
Blood in stool & 2 \\
Trace & 3 \\
Occasionally frank & \\
Usually frank & 0 \\
General well being & 1 \\
Very well & 2 \\
Slightly below par & 3 \\
Poor & 4 \\
Very poor & 1 per manifestation \\
Terrible & \\
Extracolonic features & \\
\hline
\end{tabular}

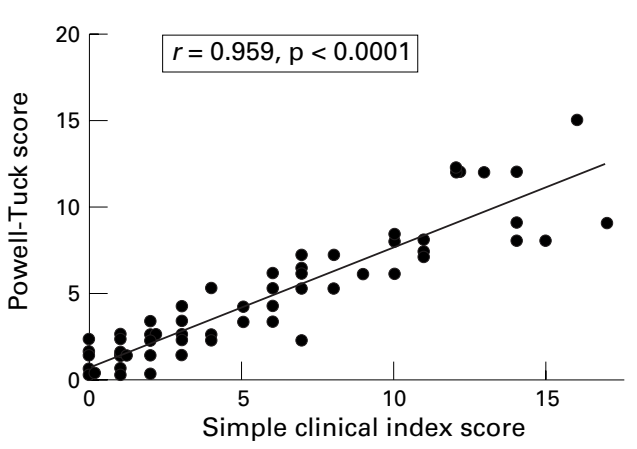

Figure 1 Scattergram showing the relation between the Powell-Tuck Index and the new Simple Clinical Colitis Activity Index ( $n=63, r=0.959, p<0.0001$, Spearman's rank correlation).

criteria and scoring system summarised in table 3 ( $r=0.959, \mathrm{p}<0.0001)$. Figure 1 shows the distribution.

The correlation coefficients between each individual question and the final score were: urgency 0.8 ; general well being 0.77 ; stool frequency (night) 0.77 ; stool frequency (day) 0.76 ; blood in stool 0.74 ; and other manifestations 0.4 .

FURTHER VERIFICATION OF THE SIMPLE CLINICAL INDEX

Sixty patients underwent a total of 113 assessments, 24 as inpatient events. Activity scores were calculated using both the Simple Clinical Colitis Activity Index and the complex index (table 3). The median age of the patients was 48.3 years (range 21.8-79.9); 34 were female. Fourteen had total/extensive disease, 28 left sided colitis, six proctosigmoiditis, and 12 proctitis alone. Eight patients were assessed through a period of relapse: two with extensive disease, four with left sided disease, and two with limited proctitis. The Simple Clinical Colitis Activity Index correlated highly significantly with the complex index $(r=0.924$, $\mathrm{p}<0.0001$; fig 2), and with the laboratory parameters (table 4 ).

Both indices mirrored closely the alteration in disease activity of the eight patients followed with relapses. Correlations between the simple 


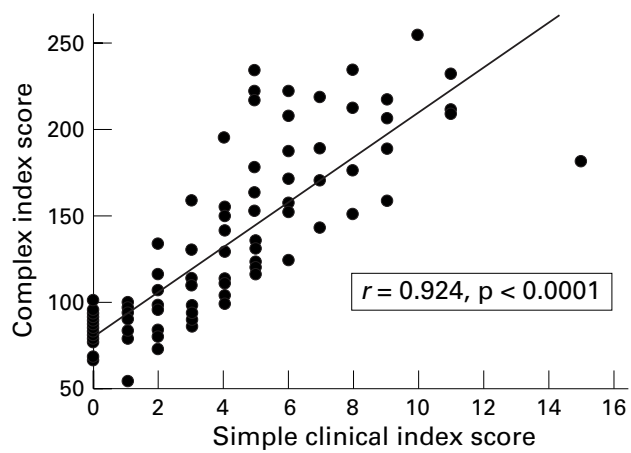

Figure 2 Scattergram showing the relation between the complex disease activity index scores and the Simple Clinical Colitis Activity Index ( $n=110, r=0.924$, $p<0.0001$, Spearman's rank correlation).

Table 4 Results of Spearman rank correlation of the Simple Clinical Colitis Activity Index score with laboratory markers

\begin{tabular}{lrrr}
\hline Laboratory marker & $n$ & $r$ & \multicolumn{1}{l}{$p$} \\
\hline Albumin & 108 & -0.607 & $<0.0001$ \\
Haemoglobin & 113 & -0.585 & 0.0002 \\
Platelet count & 113 & -0.585 & 0.0002 \\
Haematocrit & 113 & -0.575 & 0.0003 \\
Erythrocyte sedimentation rate & 98 & 0.615 & 0.0001 \\
\hline
\end{tabular}

index and both indices remained significant for all disease sites (table 5).

\section{Discussion}

The first qualitative grading of ulcerative colitis activity was introduced by Truelove and Witts ${ }^{11}$ using the five criteria of temperature, pulse rate, haemoglobin, erythrocyte sedimentation rate, and bowel frequency. Disease activity was categorised as mild, moderate, or severe so that considerable changes could occur in disease activity without a change in category. ${ }^{8}$ PowellTuck et al developed their index for clinical trials of corticosteroid treatment to include a wider range of symptoms, examination for abdominal tenderness, and sigmoidoscopic scoring systems developed by Baron and colleagues ${ }^{6}$ together with an assessment of the patient's general health. The importance of including a self assessment of the patient's own health has been shown by Drossman et al in developing a health status scale for ulcerative colitis based on an extensive questionnaire circulated to nearly one thousand members of the Crohn's and Colitis Foundation of America. ${ }^{12}$

Assessment of the macroscopic sigmoidoscopic appearance is subjective. Indeed Gomes et al found very little correlation between the macroscopic changes at colonoscopy in 28 patients and an expanded Powell-Tuck score. ${ }^{13}$

Table 5 Results of Spearman rank correlation of the Simple Clinical Colitis Activity Index score with the Powell-Tuck and complex activity index scores segregated by disease extent

\begin{tabular}{lccccccc}
\hline & \multicolumn{2}{c}{ Simple score v Powell-Tuck index } & & \multicolumn{3}{c}{ Simple score v complex index } \\
\cline { 2 - 3 } Disease site & $n$ & $r$ & $p$ & & & & \\
\hline Total & 25 & 0.95 & $<0.0001$ & & 30 & 0.92 & $<0.0001$ \\
Left sided & 17 & 0.96 & $<0.0001$ & & 37 & 0.94 & $<0.0001$ \\
Rectosigmoid & 9 & 0.8 & 0.06 & & 10 & 0.84 & 0.02 \\
Rectum & 12 & 0.97 & $<0.0001$ & & 19 & 0.88 & $<0.0001$ \\
Distal & 21 & 0.92 & $<0.0001$ & & 29 & 0.87 & $<0.0001$ \\
\hline
\end{tabular}

Distal disease is taken as rectal and rectosigmoid cases combined.
This suggests that sigmoidoscopy is not the most accurate method of assessing disease activity once the diagnosis of ulcerative colitis has been established. The ultimate aim of treatment is to reduce mucosal inflammation and the simple index still has some degree of bias towards subjective components. Histological changes will continue to play a role in the overall picture, especially of patients who do not settle as expected.

The physical examination, although reassuring to both patient and doctor is, in practice, of limited value in the initial assessment of severity and is also impractical for an index which could possibly be calculated by self assessment. To improve the symptomatic evaluation we included a separate score for the frequency of bowel motion at night as studies by Rao and coworkers $^{4}$ confirmed that nocturnal defecation and urgency of defecations are both significantly increased in active proctitis and colitis. These categories were further refined into the need to hurry to the toilet, a more severe sense of urgency, and actual incontinence.

Blood tests are important in the assessment of severe disease, but haematological and biochemical investigations are not instantly available even in the hospital setting and are inappropriate for immediate evaluation. We therefore attempted to obtain an immediate score without the use of laboratory indices.

While a complete assessment of the severity of the severely ill individual with ulcerative colitis must include symptoms, physical signs, biochemical and haematological criteria, with microscopic and macroscopic mucosal grading, activity indices incorporating all of these criteria are cumbersome to use in practice, ${ }^{7} 10121415$ and are not available until the laboratory results have been reported. We have therefore devised a simple clinically based index and compared it with an established index which involves physical examination and sigmoidoscopic assessment, a complex index utilising clinical and laboratory data, and with five haematological and biochemical markers of disease severity.

The close correlation between the indices as well as the laboratory data, suggests that the Simple Clinical Colitis Activity Index can be reliably used as an initial assessment in outpatients, by non-specialist physicians and possibly by patients themselves, as a guide to modifying treatment and the need to seek further medical advice.

1 Sinclair TS, Brunt PW, Mowat NAG. Non specific proctocolitis in North-eastern Scotland: a community based study. Gastroenterology 1983;85:1-11.

2 Devlin HB, Datta D, Dellipiani AW. The incidence and prevalence of inflammatory bowel disease in North Tees Health District. World f Surg 1980;4:183-93.

3 Powell-Tuck J, Brown RL, Lennard-Jones JE. A comparison of oral prednisolone given as a single or multiple daily doses for active proctocolitis. Scand $\mathcal{F}$ Gastroenterol 1978;13:8337.

4 Rao SSC, Holdsworth CD, Read NW. Symptoms and stool patterns in patients with ulcerative colitis. Gut 1988;29: 342-5.

5 Harvey RF, Bradshaw JM. A simple index of Crohn's disease activity. Lancet 1980;i:514.

6 Baron JH, Connell AM, Lennard-Jones JE. Variation between observers in describing mucosal appearances in proctocolitis. BMF 1964;1:89-92. 
7 Seo M, Okada M, Yao T, et al. An index of disease activity in patients with ulcerative colitis. Am $\mathcal{F}$ Gastroenterol 1992;87: 971-6.

8 Seo M, Okada M, Yao T, et al. Evaluation of disease activity in patients with moderately active ulcerative colitis comparison of a new activity index and Truelove and Witts. Am f Gastroenterol 1995;90:1759-63.

9 Cooke WT, Prior P. Determining disease activity in inflammatory bowel disease. F Clin Gastroenterol 1984;6:17-25.

10 Kambe H, Yoshida T, Haraguchi Y, et al. Quantification of disease activity in patients with ulcerative colitis. $\mathcal{f}$ Clin Gastroenterol 1986;8:651-4.

11 Truelove SC, Witts LJ. Cortisone in ulcerative colitis. Final report on a therapeutic trial. BMF 1955;2:1041-8
12 Drossman DA, Li Z, Leserman J, et al. Ulcerative colitis and Crohn's disease health status scales for research and clinical practice. F Clin Gastroenterol 1992;15:104-12.

13 Gomes P, Du Boulay C, Smith CL, et al. Relationship between disease activity indices and colonoscopic findings in patients with colonic inflammatory bowel disease. Gut 1986;27:92-7.

14 Rachmilewitz D. Coated mesalazine (5-amino-salicylic acid) versus sulphasalazine in the treatment of active ulcerative colitis: a randomised trial. BMF 1989;298:82-6.

15 Rutegard I, Ahsgren L, Stenling R, et al. A simple index for assessment of disease activity in patients with ulcerative colitis. Hepatogastroenterology 1990;37(suppl 2):110-12. 